\title{
Preparação e caracterização de scaffolds de $\beta$-fosfato tricálcico pelo método de freeze casting
}

\section{(Preparation and characterization of $\beta$-tricalcium phosphate scaffolds by freeze casting method)}

\author{
C. G. de Paula ${ }^{1}$ E. S. Trichês ${ }^{1 *}$ \\ ${ }^{1}$ Universidade Federal de São Paulo, Instituto de Ciência e Tecnologia, Laboratório de Biocerâmicas, R. Talim \\ 330, 12231-280, São José dos Campos, SP, Brasil
}

\begin{abstract}
Resumo
Scaffolds são substratos tridimensionais que permitem a diferenciação, proliferação e crescimento celular. A fim de se atingir propriedades como biocompatibilidade, adequada porosidade e interconectividade, ideais taxas de degradação e propriedades mecânicas, este estudo teve como objetivo a produção de scaffolds de $\beta$-fosfato tricálcico ( $\beta$-TCP) pelo método de freeze casting. Por meio da variação do teor de sólidos da suspensão cerâmica foi possível avaliar sua influência na microestrutura e propriedades físicas e mecânicas dos scaffolds de $\beta$-TCP. A porosidade variou entre 85 e $55 \%$ para teores de sólidos de 10 e $30 \mathrm{vol} \%$, respectivamente. A morfologia dos poros foi caracterizada por um padrão lamelar, semelhante ao formato dos cristais de gelo. O aumento do teor de sólidos na suspensão cerâmica resultou na redução do número de poros lamelares. Os valores de resistência mecânica à compressão variaram entre 0,10 e 0,71 MPa. Os scaffolds com maior valor de resistência à compressão foram submetidos ao ensaio de degradação, cujos resultados revelaram que a perda de massa dos scaffolds aumentou com tempo de imersão. O maior valor de perda de massa foi de $2,89 \pm 0,64 \%$ para período de 28 dias. Os scaffolds de $\beta$-TCP apresentam grande potencial para uso na engenharia tecidual.
\end{abstract}

Palavras-chave: freeze casting, scaffolds, engenharia tecidual, $\beta$-fosfato tricálcico.

\begin{abstract}
Scaffolds are three-dimensional substrates that allow cell differentiation, proliferation and growth. In order to achieve properties such as biocompatibility, adequate porosity and interconnectivity, ideal rates of degradation and mechanical properties, the main objective of this study was the production of $\beta$-tricalcium phosphate $(\beta-T C P)$ scaffolds by freeze casting method. Through the variation of the solids content, it was possible to evaluate its influence on the microstructure and the physical and mechanical properties of the $\beta$-TCP scaffolds. The porosity varied between 85 and $55 \%$ for solids contents of 10 and 30 vol\%, respectively. The pore morphology was characterized by a lamellar pattern, similar to ice crystals. By increasing solid content in the ceramic suspension, it was possible to observe a reduction of the number of lamellar pores. Mechanical compressive strength values ranged from 0.10 to $0.71 \mathrm{MPa}$. The scaffolds that presented the highest compressive strength value were characterized by degradation test. The results revealed that the weight loss increased with the immersion time. The highest value of the weight loss was $2.9 \pm 0.64 \%$ for the period of 28 days. The $\beta$-TCP scaffolds show great potential to use in tissue engineering.
\end{abstract}

Keywords: freeze casting, scaffolds, tissue engineering, $\beta$-tricalcium phosphate.

\section{INTRODUÇÃO}

Scaffolds são as principais ferramentas utilizadas pela engenharia tecidual para a reconstrução de tecidos. Eles podem ser definidos como substratos tridimensionais capazes de promover estabilidade mecânica necessária para a formação do tecido desejado com as funções apropriadas. É desejável que os scaffolds sejam capazes de facilitar os processos críticos no reparo dos tecidos, como a proliferação, diferenciação das células e crescimento tecidual, além de estimular os processos de reparação específicos de cada tipo de tecido [1-7]. A porosidade,

*eliandra.sousa@unifesp.br o tamanho de poros e a interconectividade também são características fundamentais, uma vez que influenciam nas interações celulares e nos processos de transporte de massa que são essenciais para o crescimento e sobrevivência das células. No caso de scaffolds para tratamento ósseo, esperase a presença de poros com diâmetro de no mínimo $100 \mu \mathrm{m}$ $[1,2]$ e interconectados, capazes de maximizar a utilização dos poros e da área superficial. A geometria dos poros por sua vez influencia na organização celular, afetando o crescimento tecidual.

Nas últimas décadas, diversos métodos vêm sendo estudados para a produção de scaffolds a fim de se garantir as propriedades físicas e mecânicas desejadas. Os métodos mais utilizados são: método da réplica [8-10]; 
incorporação de materiais orgânicos que se volatilizam durante a queima [11, 12]; método de gelcasting e emulsão [13-18]; prototipagem rápida e impressão 3D (3DP) [1921]; e freeze casting [11, 22-27]. Dentre esses destaca-se o método de freeze casting que consiste no congelamento de uma suspensão cerâmica líquida (aquosa ou não). Durante o congelamento, as partículas da suspensão são rejeitadas pela movimentação e crescimento dos cristais de solvente que estão sendo solidificados, se empacotando ao redor dos mesmos. Em seguida, ocorre a sublimação da fase solidificada a baixas temperaturas e pressões e, por fim, o corpo cerâmico é submetido à sinterização obtendo-se assim sua consolidação e densificação, resultando em uma estrutura porosa na qual os poros são réplicas dos cristais do solvente utilizado [28-31]. Existem diversas variáveis de processamento que podem ser alteradas para a obtenção de propriedades específicas, tais como: escolha do solvente que influencia na temperatura de preparo da suspensão e também na microestrutura final; condições de solidificação, como temperatura de congelamento e escolha do molde; e condições de sublimação a fim de se eliminar os cristais e assim gerar a porosidade do corpo a verde [28-31]. A formulação da suspensão cerâmica, principalmente o teor de sólidos, também influencia na morfologia, tamanho e na orientação da porosidade dos scaffolds cerâmicos. O uso da água como solvente, por exemplo, resulta em uma microestrutura lamelar, semelhante à estrutura porosa dos ossos [29, 30], com canais lamelares entre as paredes cerâmicas devido ao crescimento e à cristalografia dos cristais de gelo. Outras variáveis de processo significativas são o tipo do ligante e aditivos utilizados, que influenciam diretamente no processo de solidificação e indiretamente na interconectividade e morfologia dos poros e garantem elevada versatilidade ao método [31]. As propriedades mecânicas dos scaffolds obtidos por freeze casting, muito importantes para aplicação como biomateriais, são influenciadas principalmente por: natureza do material; dimensões dos poros e interconectividade; direção e morfologia dos poros; e integridade da estrutura. Maiores valores de resistência à compressão são atingidos quando água é utilizada como solvente, devido à formação de uma estrutura anisotrópica, ou seja, da cristalografia do gelo, que dá origem à morfologia dos poros [28, 29]. Para evitar a ocorrência de defeitos estruturais decorrentes da difusão do solvente, que geram cristais de gelo secundários, é recomendado o uso de partículas com dimensões micrométricas ou o aumento da viscosidade da suspensão, a fim de se aumentar os valores de resistência à compressão [29-32].

Para o tratamento de tecidos ósseos, destaca-se o uso das biocerâmicas, devido à semelhança de suas composições mineralógicas às destes tecidos [2, 3, 6] sendo as mais utilizadas as apatitas sintéticas, minerais de fosfato de cálcio, apatita derivada de corais e biovidro [2, $3,6,33]$. Fosfatos tricálcicos $\left[\mathrm{Ca}_{3}\left(\mathrm{PO}_{4}\right)_{2}\right]$ são exemplos de biocerâmica reabsorvível $[2,34,35]$ e apresentam quatro formas polimórficas: $\alpha, \beta, \gamma$ e super $\alpha[2,36]$. As fases $\alpha$ e $\beta$ são as mais utilizadas como biocerâmicas, sendo a fase $\beta$ mais estável podendo ser aplicada nas formas porosa e/ou densa, na ortopedia e odontologia [34, 36, 37]. O $\beta$-TCP, assim como outras biocerâmicas, promove a adesão, a proliferação e a diferenciação osteoblástica de células mesenquimatosas, assim como a produção de uma matriz de colágeno que sofre mineralização $[35,38]$. Além disso, sua presença favorece o processo de neovascularização [39]. Uma vez implantado, o TCP se dissolve e é substituído pelo novo tecido $[2,34]$. Sua reabsorção é causada por alguns fatores como: solubilidade do material e $\mathrm{pH}$ do meio; formação de novas fases superficiais; desintegração física do material em partículas menores devido ao ataque químico que acontece preferencialmente nos contornos de grão; e fatores biológicos, que podem influenciar nas condições do meio. A biodegradação dos fosfatos de cálcio aumenta com a área superficial e com a diminuição da cristalinidade, defeitos cristalinos e tamanhos de cristal e grãos [2]. Nesse contexto, este trabalho tem como objetivo a preparação e caracterização de scaffolds de $\beta$-fosfato tricálcico pelo método de freeze casting a fim de definir a melhor relação entre estrutura e propriedades, visando sua aplicação na engenharia tecidual.

\section{MATERIAIS E MÉTODOS}

Síntese do $\beta$-fosfato tricálcico: o pó de $\beta$-TCP foi sintetizado por reação via estado sólido a partir da mistura de carbonato de cálcio $\left(\mathrm{CaCO}_{3}\right.$, Synth) e fosfato bibásico anidro $\left(\mathrm{CaHPO}_{4}\right.$, Synth) na razão molar de 1:2. A reação é mostrada na Eq. A:

$$
\mathrm{CaCO}_{3}(\mathrm{~s})+2 \mathrm{CaHPO}_{4}(\mathrm{~s}) \rightarrow \beta-\mathrm{Ca}_{3}\left(\mathrm{PO}_{4}\right)_{2}(\mathrm{~s})+\mathrm{CO}_{2}(\mathrm{~g})+\mathrm{H}_{2} \mathrm{O}(\mathrm{v})(\mathrm{A})
$$

A mistura obtida foi calcinada a $1050{ }^{\circ} \mathrm{C}$ por $6 \mathrm{~h}$ em um forno mufla (EDG, $\left.1200{ }^{\circ} \mathrm{C}\right)$. Após essa etapa, o pó foi submetido à moagem via úmida em moinho de bolas (Marconi, MA500). Foram considerados dois tempos de moagem: 12 e $24 \mathrm{~h}$. Após a etapa de moagem, o pó de $\beta$-TCP foi seco em estufa a $80^{\circ} \mathrm{C}$ por $24 \mathrm{~h}$ e desaglomerado em almofariz de ágata.

Obtenção dos scaffolds de $\beta$-TCP pelo método de freeze casting: para obtenção dos scaffolds foram preparadas suspensões aquosas de $\beta$-TCP contendo diferentes teores de sólidos: 10, 20 e 30 vol\%, cuja nomenclatura adotada foi: SC-TCP10, SC-TCP20 e SC-TCP30, respectivamente. Como ligante foi utilizada uma solução de álcool polivinílico (PVA, Neon) 4,5\% (m/v). Para cada suspensão preparada, foi acrescentada solução de PVA 4,5\% (m/v) na proporção de 2 wt\% em função do teor de sólidos, ou seja, da massa de $\beta$-TCP. As suspensões foram dispersas com poliacrilato de amônio (Darvan C, R.T. Vanderbilt) e desaglomeradas por agitação magnética durante $1 \mathrm{~h}$. Em seguida, as suspensões foram vertidas em moldes cilíndricos de policloreto de vinila, PVC (12 $\mathrm{mm}$ de diâmetro e $40 \mathrm{~mm}$ de altura). Foi utilizado parafilm na base dos moldes de PVC a fim de evitar o vazamento da suspensão cerâmica quando vertida no molde. Os moldes foram posicionados sobre uma placa 
de Petri e, para evitar possíveis impurezas e contaminação, outra placa de Petri foi posicionada no topo dos mesmos. O congelamento ocorreu em um freezer (Consul, $-20{ }^{\circ} \mathrm{C}$ ), e as amostras foram retiradas após solidificação completa (24 h). As amostras congeladas foram transferidas para um liofilizador (Labconco, 76700) para sublimação dos cristais de gelo, sendo submetidas a $-84{ }^{\circ} \mathrm{Ce} 0,070$ mbar por $24 \mathrm{~h}$. Os scaffolds foram queimados ao ar, com taxa de aquecimento inicial de $2{ }^{\circ} \mathrm{C} / \mathrm{min}$ até $300{ }^{\circ} \mathrm{C}$, na qual permaneceram por $2 \mathrm{~h}$ para eliminação dos aditivos orgânicos. Por fim, foi aplicada uma taxa de $5{ }^{\circ} \mathrm{C} / \mathrm{min}$ até a temperatura de sinterização $\left(1200^{\circ} \mathrm{C}\right)$, na qual os scaffolds permaneceram por $2 \mathrm{~h}$. O resfriamento seguiu-se inercialmente até a temperatura ambiente.

Caracterização do pó de $\beta$-fosfato tricálcico $(\beta$-TCP): as fases cristalinas presentes no pó de $\beta$-TCP obtido por reação no estado sólido foram determinadas por meio de difração de raios X (DRX, X'Pert Pro, PANalytical, X'Celerator, $\mathrm{CuK} \alpha$, filtro de Ni, 20 a $60^{\circ} 2 \theta, 0,02 \%, 45 \mathrm{kV}$ e $40 \mathrm{~mA}$ ). Para identificação das fases cristalinas formadas utilizouse o banco de dados JCPDS (Joint Committee of Powder Diffraction Standards). A distribuição e o tamanho médio de partículas do pó de $\beta$-TCP após os diferentes tempos de moagem, 12 e $24 \mathrm{~h}$, foram determinados por meio da técnica de granulometria por difração a laser, utilizando um analisador de tamanho de partículas (Cilas, 1190L). As amostras foram preparadas na forma de suspensão, contendo o pó cerâmico, água e dispersante (Darvan C, R.T. Vanderbilt). Esta suspensão foi submetida à agitação por ultrassom de ponta por $15 \mathrm{~min}$ para completa desaglomeração das partículas e posteriormente foi inserida no equipamento e a leitura foi realizada.

Caracterização dos scaffolds de $\beta$-TCP: as fases cristalinas presentes nos scaffolds de $\beta$-TCP obtidos pelo método de freeze casting também foram determinadas por meio de DRX nas mesmas condições usadas para o pó. Para caracterização morfológica, os scaffolds de $\beta$-TCP foram fraturados manualmente e a microestrutura da superfície de fratura foi analisada por microscopia eletrônica de varredura (MEV, FEI, Quanta). Os scaffolds foram recobertos com uma fina camada de ouro (Bal-Tec, MCS 010) para análise. A porosidade aparente dos scaffolds de $\beta$-TCP foi determinada usando a Eq. B. A densidade geométrica $\left(\varrho_{\text {scaffold }}\right)$ de amostras cilíndricas foi calculada usando suas dimensões (altura e diâmetro) e massa. $\mathrm{O}$ valor de densidade teórica usado para o $\beta$-TCP foi de $3,07 \mathrm{~g} / \mathrm{cm}^{3}\left(\mathrm{Q}_{t}\right)$, valor retirado da carta padrão de difração de raios X ( $\beta$-TCP, JCPDS 09-0169).

$$
\mathrm{P}(\%)=\left[1-\left(\frac{\rho_{\text {scalfold }}}{\rho_{\mathrm{t}}}\right)\right] .100
$$

A resistência mecânica de pelo menos 10 scaffolds $(\mathrm{h} / \mathrm{d} \geq 2)$ para cada um dos grupos estudados foi obtida por ensaio de compressão uniaxial (Emic, DL 20000, célula de carga de $5 \mathrm{kN}$ ) à temperatura ambiente com velocidade de carregamento de $0,5 \mathrm{~mm} \cdot \mathrm{min}^{-1}$. A taxa de degradação dos scaffolds de $\beta$-TCP foi determinada por meio da avaliação da perda de massa dos scaffolds imersos em solução de $\mathrm{NaCl}$
(0,9\% em massa) em função do tempo. Os scaffolds $(\mathrm{h}=12$ $\mathrm{mm}$ e $\mathrm{d}=8 \mathrm{~mm}$ ) foram previamente pesados e imersos em solução de $\mathrm{NaCl}$ a $36,5^{\circ} \mathrm{C}$ durante $7,14,21$ e 28 dias (n=5 para cada um dos períodos). Após o tempo de incubação, os scaffolds foram retirados da solução, lavados com água destilada, secos em estufa e pesados novamente. A partir dos resultados foi obtida uma curva da perda de massa em função do tempo. Este teste teve como objetivo simular as condições dos fluídos corpóreos, sem provocar a precipitação de apatita na superfície dos scaffolds.

\section{RESULTADOS E DISCUSSÃO}

Caracterização do pó de $\beta$-fosfato tricálcico $(\beta$-TCP): o difratograma de raios $\mathrm{X}$ do pó de $\beta$-TCP obtido pela reação no estado sólido é apresentado na Fig. 1. Sua análise revelou que o pó obtido foi composto unicamente pela fase cristalina $\beta$-TCP (JCPDS 09-0169), com picos característicos em $2 \theta$ de $22,20^{\circ}, 27,76^{\circ}, 31,02^{\circ}$ e $34,37^{\circ}$. Logo, o modo de preparo, a temperatura e tempo utilizados $\left(1050{ }^{\circ} \mathrm{C} / 6 \mathrm{~h}\right)$ foram adequados para a formação da fase $\beta$, já que não houve a formação da fase cristalina $\alpha$-TCP (JCPDS 09-0348).

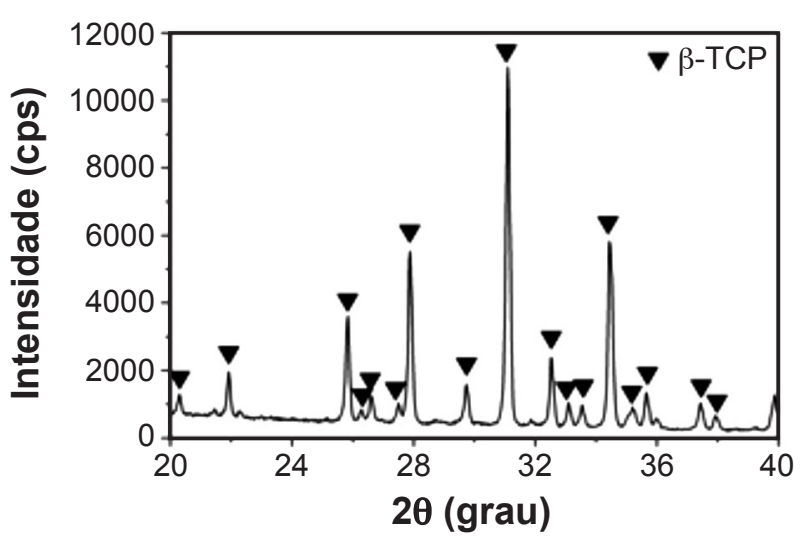

Figura 1: Difratograma de raios $\mathrm{X}$ do pó de $\beta$-TCP obtido por reação no estado sólido, $1050{ }^{\circ} \mathrm{C} / 6 \mathrm{~h}$.

[Figure 1: XDR pattern of $\beta$-TCP powder obtained by solid-state reaction, $\left.1050^{\circ} \mathrm{C} / 6 \mathrm{~h}.\right]$

A Fig. 2 apresenta as curvas de distribuição de tamanho de partículas para o pó de $\beta$-TCP obtido a partir das duas condições de moagem: 12 e $24 \mathrm{~h}$. O tamanho médio de partículas $\left(\mathrm{d}_{50}\right)$ do pó de $\beta$-TCP foi de 1,29 e 0,65 um para os tempos de moagem de 12 e $24 \mathrm{~h}$, respectivamente. Ambos os pós apresentaram distribuição monomodal de tamanho de partículas. De acordo com a literatura, o tamanho médio das partículas do pó cerâmico para utilização no método de freeze casting deve ser de ordem micrométrica, pois influencia diretamente na porosidade final dos scaffolds e resistência mecânica [29]. Estudos realizados em [32] analisaram a relação entre a estabilidade de formação dos cristais de gelo com o tamanho de partícula $(0,1$ e $10 \mu \mathrm{m})$ e a velocidade de movimentação da interface sólido/líquido em uma suspensão $\left(10^{-3}\right.$ a $\left.10^{5} \mu \mathrm{m} \cdot \mathrm{s}^{-1}\right)$. Sendo assim, a possível formação de defeitos microestruturais, semelhantes a microtrincas, está relacionada ao uso de partículas muito finas na produção 
dos corpos cerâmicos devido à instabilidade durante a solidificação das suspensões, principalmente nas regiões de interface sólido/líquido, devido à ocorrência de elevada difusividade das partículas. Além da difusividade, observase também o surgimento de cristais secundários, que são responsáveis pelo surgimento dos defeitos previamente citados, alterando a morfologia dos cristais e a distribuição das partículas durante a solidificação, podendo reduzir o comportamento mecânico à compressão [29, 32]. Portanto, para a produção dos scaffolds de $\beta$-TCP pelo método de freeze casting foi escolhido o pó de $\beta$-TCP moído por $12 \mathrm{~h}$.

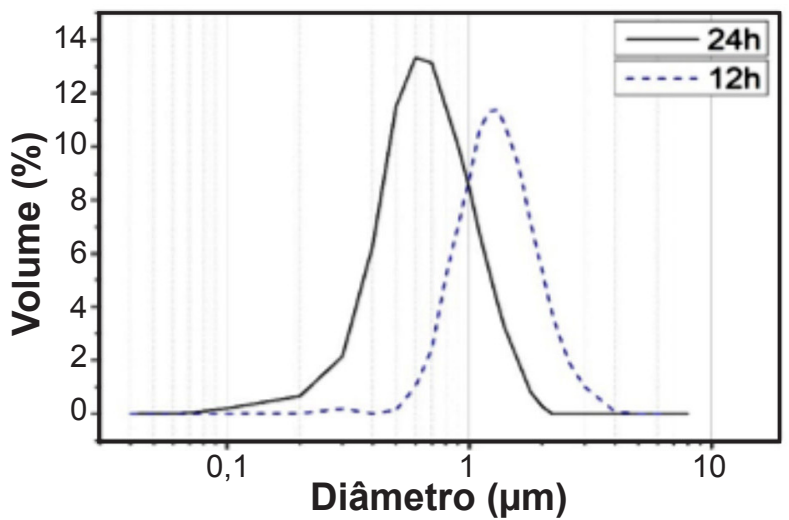

Figura 2: Curvas granulométricas do pó de $\beta$-TCP obtido por reação no estado sólido após diferentes tempos de moagem, 12 e $24 \mathrm{~h}$.

[Figure 2: Granulometric curves of $\beta$-TCP powder obtained by solid-state reaction after different milling times, 12 and $24 \mathrm{~h}$.

Caracterização dos scaffolds de $\beta$-TCP produzidos pelo método de freeze casting. Composição dos scaffolds: os difratogramas de raios $\mathrm{X}$ dos scaffolds de $\beta$-TCP preparados com as suspensões contendo 10, 20 e $30 \mathrm{vol} \%$ de sólidos são apresentados na Fig. 3. Em todas as composições foi possível identificar apenas a presença de picos característicos da fase cristalina do $\beta$-TCP (JCPDS 09-0169, $2 \theta=22,20^{\circ}$, $\left.27,76^{\circ}, 31,02^{\circ}, 34,37^{\circ}\right)$. Sendo assim, pode-se afirmar que

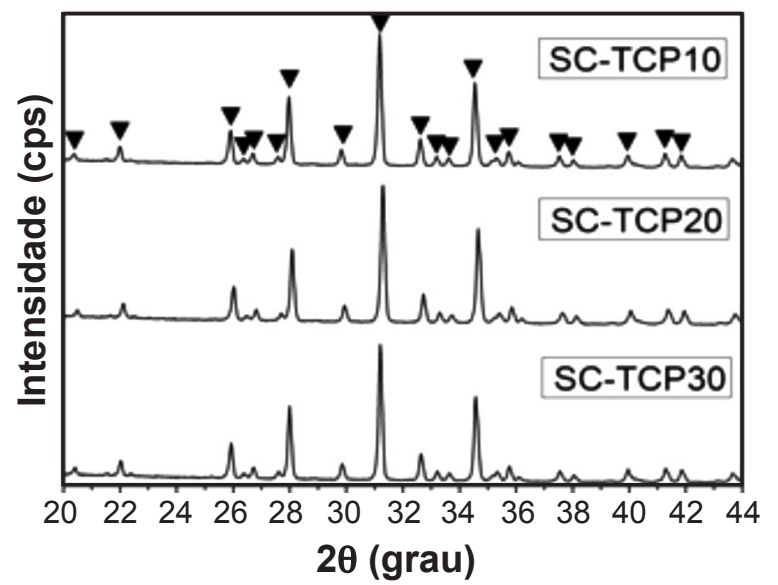

Figura 3: Difratogramas de raios $\mathrm{X}$ dos scaffolds de $\beta$-TCP sinterizados a $1200{ }^{\circ} \mathrm{C} / 2 \mathrm{~h}(\boldsymbol{\nabla}-\beta$-TCP $)$.

[Figure 3: XDR patterns of the $\beta$-TCP scaffolds sintered at $\left.1200{ }^{\circ} \mathrm{C} / 2 \mathrm{~h}.\right]$ as condições de sinterização dos scaffolds foram adequadas, uma vez que a fase $\alpha$ não foi identificada.

Morfologia dos poros: as micrografias da superfície de fratura dos scaffolds de $\beta$-TCP preparados com as suspensões contendo 10,20 e $30 \mathrm{vol} \%$ de sólidos são exibidas na Fig. 4. Foi possível avaliar que na região próxima à parede do molde os poros se formaram de maneira radial, a partir das paredes do molde e evoluíram para o centro dos scaffolds. Na região central, houve a formação dos cristais (poros) de forma multidirecional, organizados em clusters, e estes representavam o maior volume formado no interior dos scaffolds. As amostras para a caracterização microestrutural foram extraídas dessa região. Em alguns scaffolds, não houve a formação de cristais/poros na base do molde. Essas observações estão diretamente ligadas aos materiais do molde e da base utilizados na fabricação dos scaffolds de $\beta$-TCP. Logo, os poros observados apresentaram formato lamelar, semelhantes aos cristais de gelo e encontraram-se distribuídos na superfície da fratura de forma multidirecional. A microestrutura formada se assemelhou à estrutura cortical óssea, cuja porosidade é orientada na forma lamelar e o espaçamento varia entre 10 e $50 \mu \mathrm{m}$ [30]. Mesmo com algumas variações no processo de freeze casting, como uso de outras biocerâmicas [22, 26] e controle da velocidade de resfriamento [23], estudos que utilizaram água como solvente obtiveram morfologias similares às observadas neste trabalho. Foi possível constatar também que, qualitativamente, o aumento do teor de sólidos resultou no aumento da espessura das paredes e dos poros (espaçamento entre as lamelas) e na redução do número de poros na microestrutura.

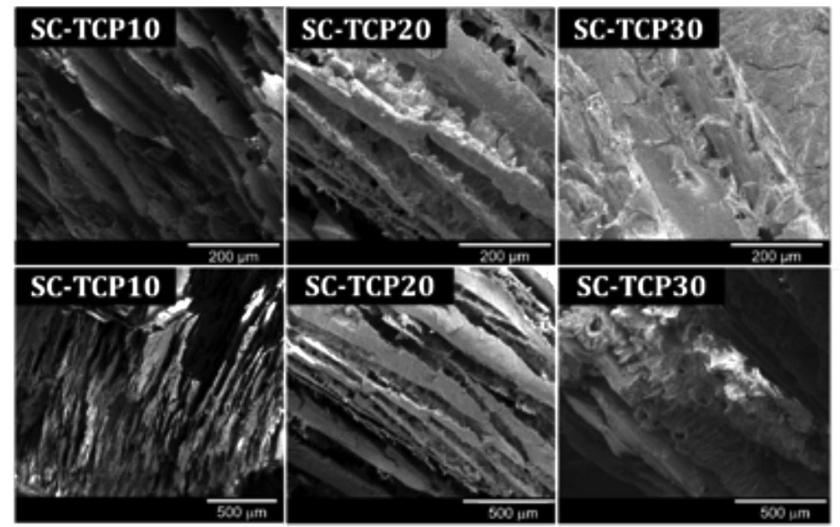

Figura 4: Micrografias de MEV dos scaffolds de $\beta$-TCP preparados pelo método de freeze casting a partir de suspensões contendo diferentes teores de sólidos: 10 vol\% (SC-TCP10); 20 vol\% (SCTCP20); e 30 vol\% (SC-TCP30).

[Figure 4: SEM micrographs of the $\beta$-TCP scaffolds prepared by freeze casting method from suspensions containing different solids content: 10 vol\% (SC-TCP10); 20 vol\% (SC-TCP20); and 30 vol\% (SC-TCP30).]

Porosidade e propriedades mecânicas: os valores de porosidade e resistência mecânica para os scaffolds de $\beta$-TCP são mostrados na Tabela I. A porosidade dos scaffolds de $\beta$-TCP preparados com a suspensão de 
10, 20 e $30 \%$ foi de $84,9 \pm 1,2,67,0 \pm 1,2$ e $55,8 \pm 2,6 \%$, respectivamente. Observou-se que o aumento do teor de sólidos da suspensão cerâmica causou uma diminuição na porosidade dos scaffolds de $\beta$-TCP. Por outro lado, a redução da porosidade causou aumento na resistência mecânica à compressão dos scaffolds, 0,10 $\pm 0,02$, $0,30 \pm 0,13$ e $0,71 \pm 0,12 \mathrm{MPa}$ para as porosidades de 84,9, 67,0 e $55,8 \%$, respectivamente. Esse comportamento foi observado em outros trabalhos [23-27, 40] e, também, era esperado, pois se sabe que porosidade e resistência mecânica são propriedades inversamente proporcionais [2]. Resultados semelhantes foram encontrados na literatura, como por exemplo o estudo [24], no qual scaffolds de $\beta$-TCP foram produzidos pelo método de freeze casting e apresentaram porosidade de $54 \%$ e resistência à compressão de aproximadamente $0,85 \mathrm{MPa}$. Flauder et al. [23], por sua vez, também produziram scaffolds de $\beta$-TCP por freeze casting, os quais apresentaram $82 \%$ de porosidade e resistência à compressão de 0,36 e 1,05 MPa para diferentes taxas de resfriamento. No entanto, deve-se ressaltar que a comparação com os resultados da literatura não é adequada, uma vez que diferentes condições de solidificação e liofilização, controle nas taxas específicas de resfriamento e de sublimação, assim como a utilização de moldes e ligantes diferentes, afetam diretamente as propriedades finais dos scaffolds. Outro ponto importante que deve ser considerado é a direcionalidade dos poros. Uma vez que a porosidade dos scaffolds foi caracterizada como predominantemente multidirecional, os resultados do ensaio mecânico indicaram que o sentido axial teve baixa incidência, caso contrário, poderia ter sido observada maior resistência mecânica à compressão na direção estudada.

Tabela I - Valores de porosidade e de resistência mecânica dos scaffolds de $\beta$-TCP.

[Table I - Values of the porosity and compressive strength of the $\beta$-TCP scaffolds.]

\begin{tabular}{ccc}
\hline Scaffold & $\begin{array}{c}\text { Porosidade } \\
(\%)\end{array}$ & $\begin{array}{c}\text { Resistência } \\
\text { mecânica } \\
(\mathrm{MPa})\end{array}$ \\
\hline SC-TCP10 & $84,9 \pm 1,2$ & $0,10 \pm 0,02$ \\
SC-TCP20 & $67,0 \pm 1,2$ & $0,30 \pm 0,13$ \\
SC-TCP30 & $55,8 \pm 2,6$ & $0,71 \pm 0,12$ \\
\hline
\end{tabular}

Para melhorar os resultados obtidos nesse trabalho, e visando a otimização dos parâmetros de processo, aliado à redução do custo, sugere-se para estudos futuros as seguintes mudanças: uso de moldes de metal (cobre ou alumínio) a fim de se aumentar a taxa de resfriamento devido à maior condutividade térmica dos materiais metálicos em comparação aos moldes de PVC e a base de vidro utilizados nesse estudo; menores temperaturas de solidificação, ou seja, utilização de temperaturas menores que $-20{ }^{\circ} \mathrm{C}$ a fim de alterar também a taxa de resfriamento devido ao $\Delta \mathrm{T}$ em relação à temperatura de produção da suspensão cerâmica; e uso de outros aditivos de processamento, que alterem a viscosidade da suspensão cerâmica. A taxa de resfriamento e a temperatura de solidificação podem atuar de forma significativa nas taxas de nucleação e crescimento dos cristais do solvente utilizado na preparação da suspensão cerâmica e, como consequência, na morfologia e nas propriedades mecânicas dos scaffolds de $\beta$-TCP [28-31].

Determinação das taxas de degradação: a avaliação da perda de massa em função do tempo foi realizada para o scaffold de $\beta$-TCP com melhor desempenho mecânico, ou seja, para o scaffold preparado com a suspensão de 30 vol\% de sólidos (SC-TCP30), Fig. 5. É possível observar que a perda de massa aumentou lentamente e de forma aproximadamente linear, e que quanto maior o tempo de degradação, maior foi a perda de massa. O valor máximo de perda de massa foi de $2,89 \pm 0,64 \%$ para o tempo de imersão de 28 dias. De acordo com a literatura, o $\beta$-TCP apresenta ótimas características de solubilidade em água, o que o torna uma das biocerâmicas mais indicadas para aplicação na engenharia tecidual, devido à possibilidade de rápida e adequada substituição pelo tecido ósseo em crescimento [37]. Resultados semelhantes foram encontrados em [41], onde a perda de massa para fosfato de cálcio poroso foi em torno de $6 \%$ para os 90 dias de imersão.

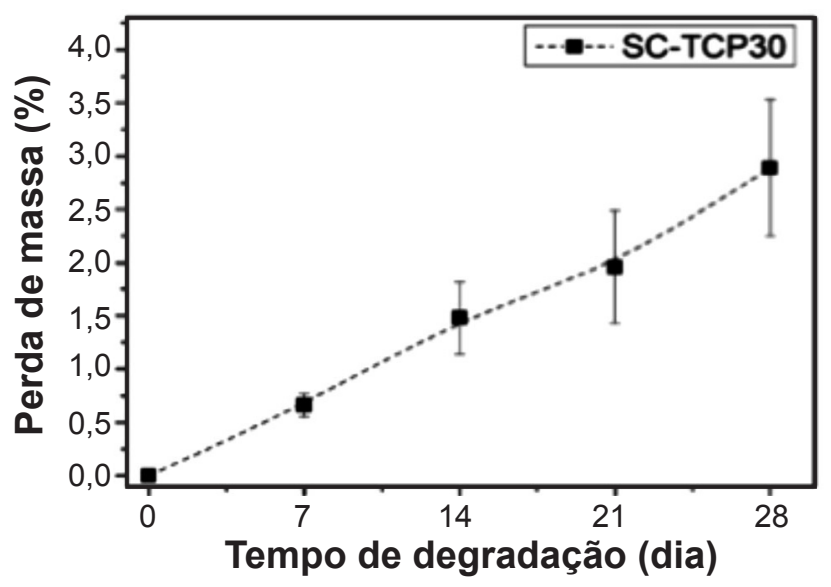

Figura 5: Taxa de degradação dos scaffolds de $\beta$-TCP preparados com a suspensão contendo 30 vol\% de sólidos (SC-TCP30).

[Figure 5: Degradation rate of $\beta$-TCP scaffolds prepared with slurry containing 30 vol\% solids (SC-TCP30).]

\section{CONCLUSÕES}

Os scaffolds de $\beta$-TCP foram produzidos com sucesso pelo método de freeze casting. A porosidade dos scaffolds variou entre 55,8 e $84,9 \%$, e os poros apresentaram formato lamelar e estavam distribuídos de forma multidirecional. O aumento do teor de sólidos da suspensão cerâmica reduziu a porosidade dos scaffolds e consequentemente ocasionou um aumento na sua resistência mecânica. Os scaffolds preparados com a suspensão contendo $30 \mathrm{vol} \%$ de sólidos apresentou o valor máximo de resistência à compressão de $0,71 \pm 0,12$ MPa, cuja porosidade foi de $55,8 \pm 2,6 \%$. A perda de massa 
dos scaffolds SC-TCP30 ocorreu de forma linear e o valor máximo foi atingido após 28 dias de imersão $(2,89 \pm 0,64 \%)$.

\section{AGRADECIMENTOS}

Os autores agradecem ao apoio da Fundação de Amparo à Pesquisa do Estado de São Paulo (Processo FAPESP 2015/24659-7) e do Conselho Nacional de Desenvolvimento Científico e Tecnológico (CNPq - Bolsa PIBITI).

\section{REFERÊNCIAS}

[1] S. Bose, M. Roy, A. Bandyopadhyay, Trends Biotechnol. 30, 10 (2012) 546.

[2]B. Ratner,A.Hoffman,F.Schoen, J.Lemons, Biomaterials science: an introduction to materials in medicine, Elsevier, Canadá (2013).

[3] C. Gao, Y. Deng, P. Feng, Z. Mao, P. Li, B. Yang, J. Deng, Y. Cao, C. Shuai, S. Peng, Int. J. Mol. Sci. 15 (2014) 4714. [4] S. Wu, X. Liu, K.W.K. Yeung, C. Liu, X. Yang, Mater. Sci. Eng. R Rep. 80 (2014) 1.

[5] R.S. Katari, A. Peloso, G. Orlando, Adv. Surg. 48, 1 (2014) 137.

[6] M. Manzano, A. Vallet-Regí, Prog. Solid State Chem. 40 (2012) 17.

[7] B.M. Holzapfel, J.C. Reichert, J.-T. Schantz, U. Gbureck, L. Rackwitz, U. Nöth, F. Jakob, M. Rudert, J. Groll, D.W. Hutmacher, Adv. Drug Deliv. Rev. 65, 4 (2013) 581.

[8] E. Sousa, C.B. Silveira, T. Fey, P. Greil, D. Hotza, A.P. Novaes de Oliveira, Adv. Appl. Ceram. 104, 1 (2005) 22.

[9] J.-H. Sung, K.-H. Shin, Y.-W. Moon, Y.-H. Koh, W.-Y. Choi, H.-E. Kim, Ceram. Int. 38 (2012) 93.

[10] S.K. Padmanabhan, F. Gervaso, M. Carrozzo, F. Scalera, A. Sannino, A. Licciulli, Ceram. Int. 39 (2013) 619. [11] E. Meurice, F. Bouchart, J.C. Hornez, A. Leriche, D. Hautcoeur, V. Lardot, F. Cambier, M.H. Fernandes, F. Monteiro, J. Eur. Ceram. Soc. 36 (2016) 2895.

[12] M. Descamps, T. Duhoo, F. Monchau, J. Lu, P. Hardouin, J.C. Hornez, A. Leriche, J. Eur. Ceram. Soc. 28, 1 (2008) 149.

[13] L. Siqueira, C.G. de Paula, M. Motisuke, R.F. Gouveia, S.E.A. Camargo, N.V.M. Milhan, E.S. Trichês, Mat. Res. 20, 4 (2017) 973.

[14] E. de Sousa, M. Dellú Jr, V. Pandolfelli, F. Ortega, Mater. Sci. Forum 591 (2008) 498.

[15] E. de Sousa, F.S. Ortega, V.C. Pandolfelli, Cerâmica 55, 334 (2009) 151.

[16] E. de Sousa, C.R. Rambo, F.S. Ortega, A.P.N. de
Oliveira, V.C. Pandolfelli, Cerâmica 55, 334 (2009) 157.

[17] H. Ge, G. Wang, B. Yuan, B. Dong, H. Li, Ceram. Int. 40 (2014) 11705.

[18] S. Dashn, R. Sarkar, S. Bhattacharyya, Ceram. Int. 41 (2015) 3775.

[19] H. Shao, Y. He, J. Fu, D. He, X. Yang, J. Xie, C. Yao, J. Ye, S. Xu, Z. Gou, J. Eur. Ceram. Soc. 36, 6 (2016) 1495.

[20] S. Bose, S. Vahabzadeh, A. Bandyopadhyay, Mater. Today 16, 12 (2013) 496.

[21] Y. Zhang, J. Zhu, Z. Wang, Y. Zhou, X. Zhang, J. Med. Hypoth. Ideas 9 (2015) 13.

[22] J. Chen, G. Liu, T.W. Button, Adv. Appl. Ceram. 112, 7 (2013) 436.

[23] S. Flauder, U. Gbureck, F. Müller, Acta Biomater. 10 (2014) 5148.

[24] N.A.S. Zairani, M. Jaafar, N. Ahmad, K.A. Razak, Ceram. Int. 42 (2016) 5141.

[25] K.K. Mallick, J. Am. Ceram. Soc. 92, S1 (2009) S85.

[26] S. Deville, E. Saiz, A. Tomsia, Biomaterials 27 (2006) 5480.

[27] Y. Tang, Q. Miao, S. Qiu, K. Zhao, L. Hu, J. Eur. Ceram. Soc. 34 (2014) 4077.

[28] S. Deville, Adv. Eng. Mater. 10 (2008) 155.

[29] S. Deville, Materials 3 (2010) 1913.

[30] M.M. Porter, J. McKittrick, M.A. Meyers, JOM 65, 6 (2013) 721.

[31] W.L. Li, K. Lu, J.Y. Walz, Int. Mater. Rev. 57, 1 (2013) 37.

[32] S. Deville, E. Maire, G. Bernard-Granger, A. Lasalle, A. Bogner, C. Gauthier, J. Leloup, C. Guizard, Nat. Mater. 8 (2009) 966.

[33] M. Mozafaria, F. Moztarzadeh, Ceram. Int. 40, 4 (2014) 5349.

[34] C. Carter, M. Norton, Ceramic materials: science and engineering, Springer, USA (2007).

[35] P. Rosseta, F. Deschaseaux, P. Layrolle, Orthop. Traumatol. Surg. Res. 100 (2014) S107.

[36] R. Carrodeguas, S. De Aza, Acta Biomater. 7 (2011) 3536.

[37] S. Dorozhkin, Biomaterials 31 (2010) 1465.

[38] S. Samavedi, A.R. Whittington, A.S. Goldstein, Acta Biomater. 9 (2013) 8037.

[39] Y. Chen, J. Wang, X.D. Zhu, Z.R. Tang, X. Yang, Y.F. Tan, Y.J. Fan, X.D. Zhang, Acta Biomater. 11 (2015) 435.

[40] S. Deville, S. Meille, J. Seuba, Sci. Technol. Adv. Mater. 16, 4 (2015) 1.

[41] S.J. Ding, C.W. Wang, D.C.H. Chen, H.C. Chang, Ceram. Int. 31 (2005) 691.

(Rec. 20/12/2017, Rev. 29/03/2018, Ac. 17/05/2018) 